\title{
STRUCTURE OF SOME NUMERICAL SEMIGROUP RINGS
}

\author{
PHILIPPE GIMENEZ \\ Instituto de Matemáticas de la Universidad de Valladolid (IMUVA) \\ Facultad de Ciencias, Universidad de Valladolid, 47011 Valladolid, Spain \\ E-mail: pgimenez@agt.uva.es \\ HEMA SRINIVASAN \\ Mathematics Department, University of Missouri \\ Columbia, MO 65211, USA \\ ORCID: 0000-0001-7509-8194_E-mail: SrinivasanH@missouri.edu
}

\begin{abstract}
This note is an extended version of the talk the second author gave at the ALaNT conference. We study the problem of constructing the minimal resolutions for the semigroup rings and use the resolution to extract information about invariants such as Betti numbers, Hilbert series, regularity etc. We illustrate the results using examples.
\end{abstract}

1. Notation. Consider a subset $\mathbf{a}=\left(a_{1}, \ldots a_{n}\right)$ of positive integers. Denote by $\langle\mathbf{a}\rangle$ the subsemigroup of $\mathbb{N}$ generated by a. Let $k$ be an arbitrary field. The semigroup ring $k[\mathbf{a}]$ is the ring $k\left[t^{j}, j \in\langle\mathbf{a}\rangle\right]$. If $\phi: k\left[x_{1}, \ldots, x_{n}\right] \rightarrow k[t]$ is the ring homomorphism defined by $\phi\left(x_{i}\right)=t^{a_{i}}$, then the image of $\phi$ is $k[\mathbf{a}] \simeq k\left[x_{1}, \ldots, x_{n}\right] / I(\mathbf{a})$. Here $I(\mathbf{a}):=\operatorname{ker} \phi$ and is therefore a prime ideal of height $n-1$ in $R:=k\left[x_{1}, \ldots, x_{n}\right]$. It is a weighted homogeneous binomial ideal with the weighting $\operatorname{deg} x_{i}:=a_{i}$ on $R$. It is also the defining ideal of the affine monomial curve $\mathcal{C}(\mathbf{a}) \subset \mathbb{A}_{k}^{n}$ parametrically defined by a whose coordinate ring is

2010 Mathematics Subject Classification: 13D02, 20M25, 13A02, $20 \mathrm{M} 14$.

Key words and phrases: semigroup, arithmetic sequence, gluing, minimal resolutions.

The first author was partially supported by Ministerio de Ciencia e Innovación (Spain) MTM2016-78881-P and Consejería de Educación de la Junta de Castilla y León VA128G18.

The second author acknowledges with thanks the support of MU Research Council grant during the preparation of this article.

The paper is in final form and no version of it will be published elsewhere. 
$k[\mathbf{a}]:=\operatorname{Im} \phi=k\left[t^{a_{1}}, \ldots, t^{a_{n}}\right] \simeq R / I(\mathbf{a})$. As $k[d \mathbf{a}]$ is isomorphic to $k[\mathbf{a}]$ for all integer $d \geq 1$, we may assume without loss of generality that $a_{1}, \ldots, a_{n}$ are relatively prime. The ring $k[\mathbf{a}]$ is now the semigroup ring of the numerical semigroup $\left\langle a_{1}, \ldots, a_{n}\right\rangle \subset \mathbb{N}$ generated by $a_{1}, \ldots, a_{n}$. The motivating question is to construct a minimal graded $R$-free resolution of $k[\mathbf{a}]$.

The ring $k[\mathbf{a}]$ is a Cohen-Macaulay domain of dimension one. Hence the projective dimension of $k[\mathbf{a}]$ over $R$ is $n-1$, where $n$ is the cardinality of $\mathbf{a}$. The fact that the ring $k[\mathbf{a}]$ is a complete intersection does not depend on the field $k$ by [6, Corollary 1.13]. Further, it is well-known that $k[\mathbf{a}]$ is Gorenstein if and only if the numerical semigroup $\left\langle a_{1}, \ldots, a_{n}\right\rangle \subset \mathbb{N}$ is symmetric, which does not depend either on the field $k$. We will thus say that $\mathbf{a}$ is a complete intersection (respectively Gorenstein) if the semigroup ring $k[\mathbf{a}]$ is a complete intersection (respectively Gorenstein).

When the sequence a minimally generates the semigroup, then the semigroup ring $k[\mathbf{a}]$ has embedding dimension $n$ which is equivalent to the ideal $I(\mathbf{a})$ being contained in $\left\langle x_{1}, \ldots, x_{n}\right\rangle^{2}$. We also say a is numerically independent if it is a minimal generating set for the semigroup $\langle\mathbf{a}\rangle$.

2. Principal matrices. If $\mathbf{a}=\left\{a_{1}, \ldots, a_{n}\right\}, n \geq 2$, is a set of relatively prime positive integers, there is an integer $g(\mathbf{a})$ such that $x>g(\mathbf{a}) \Longrightarrow x \in\langle\mathbf{a}\rangle$. This implies that for each $i, 1 \leq i \leq n$, there exists a multiple of $a_{i}$ that belongs to the numerical semigroup generated by the rest of the elements in the sequence. Denote by $r_{i}>0$ the smallest positive integer such that $r_{i} a_{i} \in\left\langle a_{1}, \ldots, a_{i-1}, a_{i+1}, \ldots, a_{n}\right\rangle$. So we have

$$
\forall i, 1 \leq i \leq n: \quad r_{i} a_{i}=\sum_{j \neq i} r_{i j} a_{j}, \quad r_{i j} \geq 0, \quad r_{i}>0 .
$$

Definition 1. The $n \times n$ matrix $D(\mathbf{a}):=\left(r_{i j}\right)$, where $r_{i i}:=-r_{i}$, is called a principal matrix associated to a.

The principal matrix $D(\mathbf{a})$ is not uniquely defined. Although the diagonal entries $-r_{i}$ are uniquely determined, there is not a unique choice for $r_{i j}$ in general. We have the "map" $D: \mathbb{N}^{[n]} \rightarrow T_{n}^{*}$ from the set $\mathbb{N}^{[n]}$ of sequences of $n$ relatively prime positive integers to the subset $T_{n}^{*}$ of $n \times n$ singular matrices with negative integers on the diagonal and nonnegative integers outside the diagonal. When $D(\mathbf{a})$ has rank $n-1$, the maximum possible, we can recover a from $D(\mathbf{a})$ by factoring out the greatest common divisor of the $n$ maximal minors of the $(n-1) \times(n-1)$ submatrix of $D(\mathbf{a})$ obtained by removing the first row. In other words, call $D^{-1}: T_{n}^{*} \rightarrow N^{[n]}$ the operation that, for $M \in T_{n}$, takes the first column of $\operatorname{adj}(M)$ and then factors out the g.c.d. to get an element in $N^{[n]}$. Given a matrix $M \in T_{n}, D\left(D^{-1}(M)\right) \neq M$ in general as the following example shows:

EXAMPLE 2.

$$
M=\left[\begin{array}{cccc}
-5 & 0 & 1 & 1 \\
0 & -3 & 1 & 1 \\
2 & 1 & -3 & 1 \\
4 & 1 & 0 & -2
\end{array}\right]
$$

The matrix $M$ has rank 3 . We see that $D^{-1}(M)=(6,10,13,17)$ and $D\left(D^{-1}(M)\right) \neq M$. It is easy to check, for example, that $r_{3}=2<3$ for $2 \cdot 13=6+2 \cdot 10$. 
3. Principal matrices and Gorenstein sequences of length 4. In this section, we denote by $E_{i j}$ the matrix all of whose entries are zeros except for the entry in the $i$ th row and $j$ th column which is 1 . The size of the matrix will be clear from the context.

Let $\mathbf{a}=\left(a_{1}, a_{2}, a_{3}, a_{4}\right)$.

If $\mathbf{a}$ is Gorenstein but is not a complete intersection, by the characterization in [1, Theorems 3 and 5], there is a permutation of a which has principal matrix $D(\mathbf{a})$ in the following form:

$$
\left[\begin{array}{cccc}
-c_{1} & 0 & d_{13} & d_{14} \\
d_{21} & -c_{2} & 0 & d_{24} \\
d_{31} & d_{32} & -c_{3} & 0 \\
0 & d_{42} & d_{43} & -c_{4}
\end{array}\right]
$$

with $c_{i} \geq 2$ and $d_{i j}>0$ for all $1 \leq i, j \leq 4$, the columns summing to zero and all the columns of the adjoint being relatively prime. The first column of the adjoint of this matrix is $-\mathbf{a}^{T}$ and Bresinsky's characterization also says that the first column of the adjoint of every such matrix defines a Gorenstein monomial curve (after removing the signs).

Definition 3. We say that a $4 \times 4$ matrix with integer entries $A=\left(a_{i j}\right)$ is pseudoGorenstein if

(1) the columns add up to zero;

(2) the entries on the diagonal are all negative;

(3) the other entries are all nonnegative;

(4) there are exactly four entries that are zero: $a_{12}=a_{23}=a_{34}=a_{41}=0$.

REMARK 4. Any pseudo-Gorenstein matrix $A$ will be of rank 3 . So adj $(A)$ has rank 1 , i.e., its columns are all equal up to a multiple. Moreover, since the columns of $A$ add up to zero, the 4 columns of $\operatorname{adj}(A)$ are the same. This means that $\operatorname{adj}(A)=\mathbf{a}^{T} \times[-1-1-1-1]$ for some $\mathbf{a}=\left(a_{1}, a_{2}, a_{3}, a_{4}\right)$.

Thus, by Brezinsky, any sequence of length four in $k^{4}$ that is Gorenstein but not a complete intersection, can be permuted so that it has a principal matrix which is pseudo-Gorenstein with the four entries in the first column of the adjoint being relatively prime. In [4], we prove a slight strengthening to show that if for a pseudo-Gorenstein matrix $D$, the first column of the adjoint of $D$ are relatively prime, then it is indeed the principal matrix of a Gorenstein non-complete intersection, namely the entries in any of the columns in its adjoint, after removing the signs.

THEOREM 5 ([4]). If $A$ is pseudo-Gorenstein and has the first column of the adjoint relatively prime integers $-a_{1},-a_{2},-a_{3},-a_{4}$, then it is indeed a principal matrix of the sequence $a_{1}, a_{2}, a_{3}, a_{4}$ and it defines a Gorenstein monomial curve which is not a complete intersection.

EXAMPLE 6.

$$
A=\left[\begin{array}{cccc}
-4 & 0 & 2 & 1 \\
3 & -6 & 0 & 4 \\
1 & 5 & -5 & 0 \\
0 & 1 & 3 & -5
\end{array}\right]
$$


The adjoint of $A$ has as its first column $(-70,-87,-101,-78)$. This, by our criterion, is now a Gorenstein sequence which is not a complete intersection and $A$ is a principal matrix associated to this sequence.

However not all pseudo-Gorenstein matrices are Gorenstein as the following example shows.

EXAMPLE 7.

$$
A=\left[\begin{array}{cccc}
-8 & 0 & 4 & 2 \\
2 & -4 & 0 & 1 \\
6 & 2 & -7 & 0 \\
0 & 2 & 3 & -3
\end{array}\right]
$$

It is pseudo-Gorenstein but the columns of $\operatorname{adj}(A)$ are not relatively prime. All the columns of the adjoint are $-4 \mathbf{a}$ for $\mathbf{a}=(16,15,18,28)$. Hence this may not be Gorenstein. In fact, the principal matrix of $(16,15,18,28)$ is

$$
A=\left[\begin{array}{cccc}
-3 & 2 & 1 & 0 \\
2 & -4 & 0 & 1 \\
1 & 0 & -4 & 2 \\
0 & 2 & 3 & -3
\end{array}\right]
$$

which is not pseudo-Gorenstein! Thus $I(\mathbf{a})$ is not Gorenstein. Its resolution is

$$
0 \rightarrow R^{3} \rightarrow R^{8} \rightarrow R^{6} \rightarrow I(\mathbf{a}) \rightarrow 0 .
$$

The following result [4] gives two families of Gorenstein monomial curves in $\mathbb{A}_{k}^{4}$ by translation from a given Gorenstein curve.

THEOREM 8. Given any Gorenstein non-complete intersection sequence a of length 4, with principal matrix $D(\mathbf{a})$, there exist two vectors $\mathbf{u}$ and $\mathbf{v}$ in $\mathbb{N}^{4}$ such that for all $t \geq 0$, the sequences $(\mathbf{a}+t \mathbf{u})$ and $(\mathbf{a}+t \mathbf{v})$ also define Gorenstein non-complete intersections whenever the entries of the corresponding sequence $(\mathbf{a}+t \mathbf{u})$ for the first family and $(\mathbf{a}+t \mathbf{v})$ for the second are relatively prime. Further, their principal matrices are given by

$$
D(\mathbf{a}+t \mathbf{u})=D(\mathbf{a})+t\left(E_{13}-E_{33}+E_{31}-E_{11}\right)
$$

and

$$
D(\mathbf{a}+t \mathbf{v})=D(\mathbf{a})+t\left(E_{24}-E_{44}+E_{42}-E_{22}\right) .
$$

We can also similarly write the resolution of the semigroup rings, as in 4 . EXAMPLE 9.

$$
A=\left[\begin{array}{cccc}
-5 & 0 & 1 & 2 \\
2 & -4 & 0 & 1 \\
3 & 2 & -4 & 0 \\
0 & 2 & 3 & -3
\end{array}\right] .
$$

The sequence is $(34,33,42,64)$ is Gorenstein, non-complete intersection, with principal matrix $A$.

Here $\mathbf{u}=(10,9,10,16)$. Set $\mathbf{a}_{\mathbf{t}}=\mathbf{a}+t \mathbf{u}$. Note that for any odd value of $t$, the entries of $\mathbf{a}_{\mathbf{t}}$ are all even and hence are not relatively prime so $\mathbf{a}_{\mathbf{t}}$ is not a Gorenstein non-complete intersection sequence in this case. When $t$ is even, a common divisor to the entries of $\mathbf{a}_{\mathbf{t}}$ 
must divide $(42+10 t)-(34+10 t)=8$ so $p=2$ is the only possible prime common divisor and since $33+9 t$ is odd, the entries of $\mathbf{a}_{\mathbf{t}}$ are relatively prime. Thus, for any even value of $t$, the sequence $\mathbf{a}_{\mathbf{t}}=\mathbf{a}+t \mathbf{u}$ is Gorenstein and it is not a complete intersection. Its principal matrix is $A_{t}=\left[\begin{array}{cccc}-5-t & 0 & 1+t & 2 \\ 2 & -4 & 0 & 1 \\ 3+t & 2 & -4-t & 0 \\ 0 & 2 & 3 & -3\end{array}\right]$. Note that when $t$ is odd, it is clear from the first row of the matrix $A_{1}$ above that the last column of its adjoint is not relatively prime and hence it is not the principal matrix of a Gorenstein non-complete intersection.

If $A$ is a pseudo-Gorenstein matrix, then there is a Gorenstein ideal associated to it: considering the vector $\mathbf{a}$ as in Remark 4 and the matrix $\phi(\mathbf{a})$ in [4, p. 676], the Pfaffian ideal of its $4 \times 4$ minors is an ideal generated by 5 elements ( 4 are the ones given by the rows of the matrix $A$, the fifth comes from Bresinsky's description) whose resolution is exactly the one we described at the end of [4] and it is Gorenstein as long as the ideal has height 3 . The thing is that this ideal might not coincide with $I(\mathbf{a})$, one can just say that it is contained in $I(\mathbf{a})$. If it is equal to $I(\mathbf{a})$, then of course $I(\mathbf{a})$ will be Gorenstein and $A=D(\mathbf{a})$.

REMARK 10. By [9, Theorem 10.2.10], the Cohen-Macaulay type of $I(\mathbf{a})$ coincides with the number of elements $\ell \in \mathbb{N}$ that are not in the semigroup $S=\langle\mathbf{a}\rangle$ and such that $\ell+s \in S$ for all $s \in S$. Of course, the Frobenius number of $S$, which is the smallest integer $g(S)$ such that $x>g(S) \Longrightarrow x \in\langle\mathbf{a}\rangle$, is always such an element so $I(\mathbf{a})$ is Gorenstein if and only if it is the only one.

Question 11. Can one tell the Cohen-Macaulay type from the principal matrix or its adjoint when it is of maximal rank?

4. Arithmetic sequences. In this section, we will consider arithmetic sequences $\mathbf{a}=$ $\left(a_{1}, a_{1}+d, \ldots, a_{1}+n d\right)$ of length $(n+1)$, which are relatively prime and minimally generate the semigroup $\langle\mathbf{a}\rangle$.

In [3], we construct minimal resolutions for all these semigroup rings and derive formulae for various invariants. These form a very important class of semigroup rings.

EXAMPLE 12. Consider the arithmetic sequence $\mathbf{a}=(7,11,15,19,23,27)$. In this case, the semigroup is symmetric.

Let $\boldsymbol{F}$ be the Eagon-Northcott complex resolving the ideal $J \subset R=k\left[x_{1}, \ldots, x_{6}\right]$ of $2 \times 2$ minors of the $2 \times 5$ matrix which must be contained in $I_{A}$, namely

$$
J=I_{2}\left(\begin{array}{lllll}
x_{1} & x_{2} & x_{3} & x_{4} & x_{5} \\
x_{2} & x_{3} & x_{4} & x_{5} & x_{6}
\end{array}\right) .
$$

Then, if $\boldsymbol{F}: 0 \rightarrow F_{4} \rightarrow F_{3} \rightarrow F_{2} \rightarrow F_{1} \rightarrow F_{0}=R \rightarrow R / J \rightarrow 0$ is the resolution of $R / J$, the resolution of $R / I_{\mathbf{a}}$ is

$$
0 \rightarrow R=R^{*} \rightarrow F_{4} \oplus F_{1}^{*} \rightarrow F_{3} \oplus F_{2}^{*} \rightarrow F_{2} \oplus F_{3}^{*} \rightarrow F_{1} \oplus F_{4}^{*} \rightarrow R \rightarrow R / I_{\mathbf{a}} \rightarrow 0
$$

which is symmetric. Here $F^{*}$ denotes the dual $\operatorname{Hom}_{R}(F, R)$. 
How do we know that this semigroup is symmetric? In [3], we show the following.

THEOREM 13. When the semigroup is minimally generated by an arithmetic sequence $\mathbf{a}=\left(a_{1}, a_{1}+d, a_{1}+2 d, \ldots, a_{1}+n d\right)$, all the Betti numbers of the semigroup ring $k[\mathbf{a}]$ are determined by $a_{1}$ modulo $n$ and the Cohen-Macaulay type of the resolution is $a_{1}-1$ modulo $n$, unless when $a_{1}-1$ is a multiple of $n$ where it is $n$.

Thus, an arithmetic sequence $\left(a_{1}, a_{1}+d, \ldots, a_{1}+n d\right)$ is symmetric if $a_{1} \equiv 2$ modulo $n$. In Example 12 we have $7 \equiv 2$ modulo 5 . So, $(7,11,15,19,23,27)$ is symmetric but $(7,11,15,19,23,27,31)$ is not symmetric.

5. Gluing. We say a sequence $\mathbf{c}$ is a gluing of two relatively prime sequences $\mathbf{a}$ and $\mathbf{b}$ if $\mathbf{c}=k_{1} \mathbf{a} \cup k_{2} \mathbf{b}$ with $k_{1}$ and $k_{2}$ relatively prime and such that $k_{1} \in\langle\mathbf{b}\rangle \backslash \mathbf{b}, k_{2} \in\langle\mathbf{a}\rangle \backslash \mathbf{a}$. This is equivalent to the notion of gluing defined in [8]. The assumptions $k_{1} \notin \mathbf{b}$ and $k_{2} \notin \mathbf{a}$ are there to ensure that $C=\mathbf{c}=k_{1} \mathbf{a} \cup k_{2} \mathbf{b}$ minimally generates the semigroup $\langle\mathbf{c}\rangle$.

In [5], we construct minimal resolutions of the semigroup rings $k[\mathbf{c}]$ obtained by gluing. Let $\mathbf{a}=\left(a_{1}, \ldots, a_{p}\right), \mathbf{b}=\left(b_{1}, \ldots, b_{q}\right)$, so that $\mathbf{c}=\left(k_{1} a_{1}, \ldots, k_{1} a_{p}, k_{2} b_{1}, \ldots, k_{2} b_{q}\right)$. Setting $R_{\mathbf{a}}=k\left[x_{1}, \ldots, x_{p}\right]$ and $R_{\mathbf{b}}=k\left[y_{1}, \ldots, y_{q}\right]$, one has $k[\mathbf{a}]=R_{\mathbf{a}} / I(\mathbf{a})$ and $k[\mathbf{b}]=R_{\mathbf{b}} / I_{B}$. Then $k[\mathbf{c}]=R / I(\mathbf{c})$ where $R=k\left[x_{1}, \ldots, x_{p}, y_{1}, \ldots, y_{q}\right]=R_{\mathbf{a}} \otimes_{k} R_{\mathbf{b}}$.

We will now see what $\mathbf{c}$ being a gluing means in terms of the minimal generating set of $I(\mathbf{c})$. The following lemma collects some easy observations; see [5].

LEMMA 14. Let $\mathbf{c}=k_{1} \mathbf{a} \cup k_{2} \mathbf{b}$ be a gluing of $\mathbf{a}$ and $\mathbf{b}$. Then:

(1) If $\mathbf{a}$ and $\mathbf{b}$ are numerically independent then so is $\mathbf{c}$.

(2) Since $k_{1} \in\langle\mathbf{b}\rangle$ and $k_{2} \in\langle\mathbf{a}\rangle$, there exist nonnegative integers $\alpha_{i}$, $\beta_{j}$ such that $k_{1}=\sum_{j=1}^{q} \beta_{j} b_{j}$ and $k_{2}=\sum_{i=1}^{p} \alpha_{i} a_{i}$.

(3) The ideal $I(\mathbf{c})$ is minimally generated by the union of minimal generating sets of the ideals $I(\mathbf{a})$ and $I(\mathbf{b})$, and exactly one other element

$$
\rho=\prod_{i=1}^{p} x_{i}^{\alpha_{i}}-\prod_{j=1}^{q} y_{j}^{\beta_{j}} \in R .
$$

(4) $\rho$ is homogeneous of degree $k_{1} k_{2}$ if one gives to each variable in $R$ the corresponding weight in $\mathbf{c}=\left(k_{1} a_{1}, \ldots, k_{1} a_{p}, k_{2} b_{1}, \ldots, k_{2} b_{q}\right)$.

Now we are ready to state the theorem on resolutions.

TheOrem 15 ([5]). Suppose that $\mathbf{c}$ is a gluing of $\mathbf{a}$ and $\mathbf{b}$ with $\mathbf{c}=k_{1} A \cup k_{2} B$ and let $F_{\mathbf{a}}$ and $F_{\mathbf{b}}$ be minimal resolutions of $k[\mathbf{a}]$ and $k[\mathbf{b}]$ respectively. Then, a minimal graded free resolution of the semigroup ring $k[\mathbf{c}]$ is obtained as the mapping cone of the map of complexes $\rho: F_{\mathbf{a}} \otimes F_{\mathbf{b}} \rightarrow F_{\mathbf{a}} \otimes F_{\mathbf{b}}$, where $\rho$ is induced by multiplication by $\rho$. In particular, $\left(I(\mathbf{a}) \cdot R+I(\mathbf{b}) \cdot R:_{R} \rho\right)=I(\mathbf{a}) \cdot R+I(\mathbf{b}) \cdot R$.

EXAmPle 16. Consider the sequence $\mathbf{c}=(187,289,425,323,140,364,336)$ which is a gluing since $\mathbf{c}=k_{1} \mathbf{a} \cup k_{2} \mathbf{b}$ for $\mathbf{a}=(11,17,25,19), \mathbf{b}=(5,13,12), k_{1}=17=5+$ $12 \in\langle\mathbf{b}\rangle$ and $k_{2}=28=11+17 \in\langle\mathbf{a}\rangle$. Set $R_{\mathbf{a}}=k\left[x_{1}, \ldots, x_{4}\right], R_{\mathbf{b}}=k\left[y_{1}, y_{2}, y_{3}\right]$ and $R=k\left[x_{1}, \ldots, y_{3}\right]$. The ideal $I(\mathbf{a}) \subset R_{\mathbf{a}}$ is minimally generated by 5 binomials, 
$f_{1}=x_{1} x_{3}-x_{2} x_{4}, f_{2}=x_{4}^{4}-x_{2}^{3} x_{3}, f_{3}=x_{2}^{4}-x_{1} x_{4}^{3}, f_{4}=x_{3}^{2}-x_{1}^{3} x_{2}, f_{5}=x_{1}^{4}-x_{3} x_{4}$. The ring $k[\mathbf{a}] \simeq R_{\mathbf{a}} / I(\mathbf{a})$ is Gorenstein, and its minimal free resolution is

$$
\begin{aligned}
& \left(\begin{array}{l}
f_{1} \\
f_{2} \\
f_{3} \\
f_{4} \\
f_{5}
\end{array}\right) \quad\left(\begin{array}{rrrrr}
0 & x_{3} & x_{1}^{3} & x_{2}^{3} & x_{4}^{3} \\
-x_{3} & 0 & 0 & x_{1} & x_{2} \\
-x_{1}^{3} & 0 & 0 & x_{4} & x_{3} \\
-x_{2}^{3} & -x_{1} & -x_{4} & 0 & 0 \\
-x_{4}^{3} & -x_{2} & -x_{3} & 0 & 0
\end{array}\right) \\
& 0 \rightarrow R_{\mathbf{a}} \stackrel{\longrightarrow}{\longrightarrow} R_{\mathbf{a}}^{5} \quad \longrightarrow \quad R_{\mathbf{a}}^{5} \quad \longrightarrow \quad R_{\mathbf{a}} \rightarrow k[\mathbf{a}] \rightarrow 0 . \\
& \left(f_{1} \ldots f_{5}\right)
\end{aligned}
$$

The ideal $I(\mathbf{b}) \subset R_{\mathbf{b}}$ is generated by 3 binomials, $g_{1}=y_{1}^{5}-y_{2} y_{3}, g_{2}=y_{2}^{3}-y_{1}^{3} y_{3}^{2}$, $g_{3}=y_{3}^{3}-y_{1}^{2} y_{2}^{2}$, it is Hilbert-Burch, and the minimal free resolution of $k[\mathbf{b}] \simeq R_{\mathbf{b}} / I(\mathbf{b})$ is

$$
\begin{aligned}
& \left(\begin{array}{ll}
y_{3}^{2} & y_{2}^{2} \\
y_{1}^{2} & y_{3} \\
y_{2} & y_{1}^{3}
\end{array}\right) \\
& 0 \rightarrow R_{\mathrm{b}}^{2} \quad \longrightarrow \quad R_{\mathrm{b}}^{3} \stackrel{\longrightarrow}{\longrightarrow} R_{\mathbf{b}} \rightarrow k[\mathbf{b}] \rightarrow 0 \text {. }
\end{aligned}
$$

The tensor product of these two resolutions provides a minimal free resolution of $R / J$ where $J=I(\mathbf{a}) \cdot R+I(\mathbf{b}) \cdot R$ :

$$
0 \rightarrow R^{2} \rightarrow R^{13} \rightarrow R^{26} \rightarrow R^{22} \rightarrow R^{8} \rightarrow R \rightarrow R / J \rightarrow 0 .
$$

Note that the differentials can be easily written down if needed.

Finally, the extra minimal generator in $I(\mathbf{c})$ is $\rho=x_{1} x_{2}-y_{1} y_{3}$ and the mapping cone induced by multiplication by $\rho$ gives a minimal resolution of $k[\mathbf{c}]$ as $R$-module:

$$
0 \rightarrow R^{2} \rightarrow R^{15} \rightarrow R^{39} \rightarrow R^{48} \rightarrow R^{30} \rightarrow R^{9} \rightarrow R \rightarrow k[\mathbf{c}] \rightarrow 0 .
$$

The differentials are given by the mapping cone construction.

We obtain many corollaries from the theorem, some new and some recovering results known for semigroup rings by other methods. Using the explicit resolution, we can read off many invariants, such as Hilbert series, Cohen-Macaulay type, Betti numbers and ranks for syzygies, etc. We list all the corollaries in one statement.

Corollary 17. Suppose that $\mathbf{c}=k_{1} \mathbf{a} \cup k_{2} \mathbf{b}$ is a gluing. Then:

(1) The ith Betti number $\beta_{i}$ of $k[\mathbf{c}]$ is given, in terms of the Betti numbers of $k[\mathbf{a}]$ and $k[\mathbf{b}]$, by the formulae

$$
\forall i \geq 0: \beta_{i}=\sum_{i^{\prime}=0}^{i} \beta_{i^{\prime}}(\mathbf{a})\left[\beta_{i-i^{\prime}}(\mathbf{b})+\beta_{i-i^{\prime}-1}(\mathbf{b})\right]=\sum_{i^{\prime}=0}^{i} \beta_{i^{\prime}}(\mathbf{b})\left[\beta_{i-i^{\prime}}(\mathbf{a})+\beta_{i-i^{\prime}-1}(\mathbf{a})\right] .
$$

(2) The Cohen-Macaulay type is given by Type $(k[\mathbf{c}])=\operatorname{Type}(k[\mathbf{a}]) \operatorname{Type}(k[\mathbf{b}])$.

(3) $k[\mathbf{c}]$ is Gorenstein, respectively a complete intersection, if and only if $k[\mathbf{a}]$ and $k[\mathbf{b}]$ are both Gorenstein, respectively complete intersections.

(4) If neither $k[\mathbf{a}]$ nor $k[\mathbf{b}]$ is Gorenstein, then the Cohen-Macaulay type of $k[\mathbf{c}]$ is not prime. 
(5) The graded Betti numbers are given by the formula

$$
\beta_{i, j}(\mathbf{c})=\sum_{i^{\prime}=0}^{i}\left(\sum_{r, s / k_{1} r+k_{2} s=j} \beta_{i^{\prime} r}(\mathbf{a})\left[\beta_{i-i^{\prime}, s}(\mathbf{b})+\beta_{i-i^{\prime}-1, s-k_{1}}(\mathbf{b})\right]\right)
$$

(6) The regularity can be seen as

$$
\operatorname{reg}(k[\mathbf{c}])=k_{1} \operatorname{reg}(k[\mathbf{a}])+k_{2} \operatorname{reg}(k[\mathbf{b}])+\left(p+k_{2}-1\right)\left(q+k_{1}-1\right)-p q .
$$

(7) The Hilbert series of $k[\mathbf{c}]$ is given by $H_{\mathbf{c}}(t)=\left(1-t^{k_{1} k_{2}}\right) H_{\mathbf{a}}\left(t^{k_{1}}\right) H_{\mathbf{b}}\left(t^{k_{2}}\right)$.

(8) If the minimal free resolutions of $k[\mathbf{a}]$ and $k[\mathbf{b}]$ admit a differential graded algebra structure, then $k[\mathbf{c}]$ inherits the structure from that of $k[\mathbf{a}]$ and $k[\mathbf{b}]$. That is, we can explicitly construct a multiplication on the minimal resolution of $k[\mathbf{c}]$ if we know the multiplication on those of $k[\mathbf{a}]$ and $k[\mathbf{b}]$.

Recall that if a resolution $F$ admits a multiplication which makes it an associative, graded commutative differential graded algebra, we say it has a differential algebra structure or a DG algebra structure.

Consider Example 16. Say, we want the second Betti number. By Corollary 17, one has $\beta_{2}(\mathbf{c})=\sum_{i^{\prime}=0}^{2} \beta_{i^{\prime}}(\mathbf{b})\left[\beta_{2-i^{\prime}}(\mathbf{a})+\beta_{2-i^{\prime}-1}(\mathbf{a})\right]=\beta_{2}(\mathbf{a})+\beta_{1}(\mathbf{a}) \beta_{1}(\mathbf{b})\left(\beta_{1}(\mathbf{a})+1\right)+\beta_{2}(\mathbf{b}) \cdot 1=$ $5+5+3 \cdot 6+2=30$.

Here is another example of gluing.

EXAMPLE 18.

$$
\mathbf{c}=(450,522,576,612,305,732,793)=k_{1} \mathbf{a} \cup k_{2} \mathbf{b}
$$

with $\mathbf{a}=(25,29,32,34), \mathbf{b}=(5,12,13), k_{1}=18$ and $k_{2}=61$. The Betti numbers of $k[\mathbf{a}]$ and $k[\mathbf{b}]$ are

\begin{tabular}{|c|c|c|c|c|}
\hline$i$ & 0 & 1 & 2 & 3 \\
\hline$\beta_{i}(\mathbf{a})$ & 1 & 7 & 10 & 4 \\
\hline
\end{tabular}

\begin{tabular}{|c|c|c|c|}
\hline$i$ & 0 & 1 & 2 \\
\hline$\beta_{i}(\mathbf{b})$ & 1 & 3 & 2 \\
\hline
\end{tabular}

Applying our formula in corollary, one infers that $\beta_{0}=\beta_{0}(\mathbf{c})=1$, and

$$
\begin{aligned}
& \beta_{1}=1(3+1)+7.1=11=1(7+1)+3.1 \\
& \beta_{2}=1(2+3)+7(3+1)+10.1=43=1 .(10+7)+3(7+1)+2.1 \\
& \beta_{3}=1.2+7(2+3)+10(3+1)+4.1=81 \\
& \beta_{4}=7.2+10(2+3)+4(3+1)=80=1.4+3(4+10)+2(10+7) \\
& \beta_{5}=10.2+4(2+3)=40=3.4+2(4+10) \\
& \beta_{6}=4.2=8
\end{aligned}
$$

and the minimal free resolution of $k[\mathbf{c}]$ shows as

$$
0 \rightarrow R^{8} \rightarrow R^{40} \rightarrow R^{80} \rightarrow R^{81} \rightarrow R^{43} \rightarrow R^{11} \rightarrow R \rightarrow k[\mathbf{c}] \rightarrow 0 .
$$

In Examples 16 and 18 above, the resolution of the minimal semigroup ring does admit a DG algebra structure. This is because both $k[\mathbf{a}]$ and $k[\mathbf{b}]$ have resolutions of length less than three and hence admit a DG algebra structure and by our corollary, the resolution of $k[\mathbf{c}]$ must admit a DG algebra structure. 


\section{References}

[1] H. Bresinsky, Symmetric semigroups of integers generated by 4 elements, Manuscripta Math. 17 (1975), 205-219.

[2] C. Delorme, Sous-monoüdes d'intersection complète de N, Ann. Sci. École Norm. Sup. (4) 9 (1976), 145-154.

[3] P. Gimenez, I. Sengupta, H. Srinivasan, Minimal graded free resolutions for monomial curves defined by arithmetic sequences, J. Algebra 388 (2013), 294-310.

[4] P. Gimenez, H. Srinivasan, A note on Gorenstein monomial curves, Bull. Braz. Math. Soc. (N.S.) 45 (2014), 671-678.

[5] P. Gimenez, H. Srinivasan, The structure of the minimal free resolution of semigroup rings obtained by gluing, J. Pure Appl. Algebra 223 (2019), 1411-1426.

[6] J. Herzog, Generators and relations of abelian semigroups and semigroup rings, Manuscripta Math. 3 (1970), 175-193.

[7] A. V. Jayanthan, H. Srinivasan, Periodic occurence of complete intersection monomial curves, Proc. Amer. Math. Soc. 141 (2013), 4199-4208.

[8] J. C. Rosales, P. A. García-Sánchez, Numerical Semigroups, Dev. Math. 20, Springer, New York, 2009.

[9] R. H. Villarreal, Monomial Algebras, Monogr. Textbooks Pure Appl. Math. 238, Marcel Dekker, New York, 2001. 
